Pacific Journal of Mathematic 


\title{
ON LEVI FACTORS OF DERIVATION ALGEBRAS AND THE RADICAL EMBEDDING PROBLEM
}

\author{
Francis J. Flanigan
}

\begin{abstract}
Problem: given a finite-dimensional nilpotent associative $k$-algebra $N$, find all unital associative $k$-algebras $A$ such that $\operatorname{rad} A=N$. An approach: which subalgebras of $\operatorname{Der}_{k} N$ are images of Lie homomorphisms $A / N \rightarrow \operatorname{Der}_{k} N$ ? Here the author constructs $N$ over very general fields $k$ such that the "Levi factor" of $\operatorname{Der}_{k} N$ is a direct sum of orthogonal Lie algebras $o(V, b)$ of arbitrarily prescribed symmetric and alternate bilinear spaces. In particular, if $k$ is algebraically closed of characteristic zero, then every direct sum of classical simple Lie algebras $A_{n}, B_{n}, C_{n}, D_{n}$ is Levi factor of some $\operatorname{Der}_{k} N$.
\end{abstract}

1. Some questions. We ask initially: What are the possible Levi factors (or semisimple semidirect summands or, more generally, semisimple subalgebras) of the Lie algebras $\operatorname{Der}_{k} N$ of all $k$-derivations $N \rightarrow N$, where $N$ is required to be a nilpotent associative algebra finite dimensional over a field $k$ (not necessarily of characteristic zero, and possibly finite)?

This rather general question, to be sharpened below, was prompted by a certain approach to the following radical embedding problem posed in Marshall Hall's [4] and in our [2], [3]:

1.1. Given a nilpotent $N$ as above, describe the set of unital associative $k$-algebras $A$ satisfying $\operatorname{rad} A=N$ (together with a certain nondegeneracy condition [2]).

The approach referred to above is this: If the scalar field $k$ is perfect, then each solution $A$ to 1.1 admits a semidirect Wedderburn decomposition $A=S+N$, with $S$ a separable semisimple subalgebra. Since $N$ is a Lie ideal in $A_{\text {Lie }}$, the usual bracketing induces a Lie homomorphism $S_{\mathrm{Lie}} \rightarrow \operatorname{Der}_{k} N$ witn "small" kernel (thanks to the nondegeneracy condition). Moreover, for reasonable fields $k$ the algebra $S_{\text {Lie }}$ is a direct sum of Lie ideals each of the form $\operatorname{sl}\left(r_{i}, k\right) \oplus k$ for various ranks $r_{i}$. We conclude that a solution $A$ to $\operatorname{rad} A=N$ in (1.1) tends to force $\operatorname{Der}_{k} N$ to contain various copies of the familiar special linear Lie algebra [3].

It was this last observation that led us ask the easily stated but far too general "survey" question of the first paragraph above. (Thus in characteristic $p>0$, there need not be a Levi-Malcev decomposition, and moreover the theory of semisimple Lie algebras is 
far from complete, even over algebraically closed fields.) In the present paper, we offer answers, adequate for our purposes, to these sharpened versions of the original question:

1.2. Is every special linear Lie subalgebra of $\operatorname{Der}_{k} N$ induced in the above manner by embedding $N$ in an associative $A=S+N$ ?

1.3. Are there nilpotent $N$ which admit orthogonal or symplectic or other "classical" Lie algebras of derivations, apparently unrelated to the radical embeddings of $N$ ?

2. Results and consequences. The point of the theorems below is the existence of nilpotent $N$ whose derivation algebras have semidirect sum decompositions with certain prescribed "wild" summands. In perticular, the answers to the questions above are: to (1.2), no (in Theorem 2.1, obtain $s l(2, k)$ as the orthogonal Lie algebra of the alternate form $\left.b\left(\left(X_{1}, X_{2}\right),\left(Y_{1}, Y_{2}\right)\right)=X_{1} Y_{2}-X_{2} Y_{1}\right)$ and, to question 1.3 , yes.

THEOREM 2.1. Given any field $k$ of characteristic not 2 and any array $\left(V_{1}, b_{1}\right), \cdots,\left(V_{m}, b_{m}\right)$ of finite-dimensional nondegenerate symmetric or alternate bilinear $k$-spaces, there exists a finitedimensional nilpotent associative k-algebra $N$ such that

(i) $N$ is directly indecomposable,

(ii) the derivation algebra $\operatorname{Der}_{k} N$ is the semidirect sum $\Lambda+\Omega$ of a Lie subalgebra $\Lambda$ and a nilpotent ideal $\Omega$,

(iii) $\Lambda \cong o\left(V_{1}, b_{1}\right) \oplus \cdots \oplus o\left(V_{m}, b_{m}\right)$,

(iv) the ideal $\Omega$ consists of nilpotent derivations.

In the above statement $o(V, b)$ is the orthogonal Lie algebra of the bilinear space $(V, b)$, that is,

$$
o(V, b)=\left\{f \in \operatorname{End}_{k} V \mid b(f(x), y)+b(x, f(y))=0 \text {, all } x, y \in V\right\} .
$$

Note that we do not assert that $o(V, b)$ is always simple.

ThEOREM 2.2. Let $k$ and $\left(V_{i}, b_{i}\right)$ be as in (2.1), and suppose given integers $r_{1}, \cdots, r_{n} \geqq 2$, none divisible by the characteristic of $k$. Then there exists a finite-dimensional nilpotent associative $k$ algebra $N$ such that

(i) the derivation algebra $\operatorname{Der}_{k} N$ is the semidirect sum $\Lambda+\Omega$ of a Lie subalgebra $\Lambda$ and a solvable ideal $\Omega$,

(ii) $\Lambda \cong\left(\bigoplus_{i=1}^{i=m} o\left(V_{i}, b_{i}\right)\right) \bigoplus\left(\bigoplus_{j=1}^{i=n} s l\left(r_{j}, k\right)\right)$,

(iii) a maximal toroidal subalgebra of $\Omega$ has dimension $n+1$.

COROLLARY 2.3. Let $k$ be an algebraically closed field of charac- 
teristic zero. Given any finite direct sum $\Sigma$ of classical simple special linear, orthogonal, and symplectic Lie algebras (types $A_{n}, B_{n}, C_{n}, D_{n}$ ) over $k$, there is a finite-dimensional nilpotent associative k-algebra $N$ such that the Levi factor of $\operatorname{Der}_{k} N$ is isomorphic to $\Sigma$.

We will construct these $N$ in $\S \S 3-5$. Here are some further comments.

2.4. In the language of [3], Theorem 2.1 produces nilpotent $N$ of genus zero, that is, nilpotents to which one can adjoin a unity element in the familiar way (cf. $A=k \cdot 1+N$ ), but cannot adjoin any more complicated semisimple $S$. The direct indecomposability is essential here. These $N$ of genus zero are noteworthy in that their derivation algebras are far from nilpotent. Compare Theorem 4.1 and the examples of $\S 5$ of [3].

2.5. Analogous constructions may be carried out for nilpotent Lie algebras $N$. In this case one may begin with the characteristically nilpotent Lie algebra of Dixmier-Lister [1] in place of the algebra $W(\S 3)$ all of whose derivations are nilpotent. A special case of such a construction, involving one split simple three-dimensional Lie algebra, was effected by Leger and Luks in [6, Proposition 6.5].

We are pleased to acknowledge a helpful correspondence with George Leger on these questions, as well as our indebtedness to certain ideas in $\S 6$ of [6].

3. Lemmas on nilpotent derivation algebras. Let the arbitrary not necessarily associative $k$-algebra $B=I_{1} \oplus \cdots \oplus I_{n}$ be a direct sum of (two-sided) ideals. We will now relate the derivation algebra of $B$ to those of the $I_{i}$. Define the subspaces

$$
\Delta_{i i}=\left\{D \in \operatorname{Der}_{k} B \mid D\left(I_{i}\right) \subset I_{i}, D\left(I_{h}\right)=0 \text { for } h \neq i\right\},
$$

and, for $i \neq j$,

$$
\Delta_{\imath j}=\left\{D \in \operatorname{Der}_{k} B \mid D\left(I_{h}\right)=D\left(I_{j}^{2}\right)=(0), h \neq j ; D\left(I_{j}\right) \subset \text { Ann } I_{i}\right\},
$$

where Ann $I_{i}$ is the two-sided annihilator of $I_{i}$ in $I_{i}$.

The following is standard. Statement (ii) is Exercise 19, page 30 of Jacobson [5].

Lemma 3.1. Let $B=I_{1} \oplus \cdots \oplus I_{n}$ as above. Then

(i) $\operatorname{Der}_{k} B=\bigoplus_{i, j} \Delta_{i j}$ as a k-space;

(ii) if each $I_{i}^{2}=I_{i}$, then $\operatorname{Der}_{k} B=\bigoplus_{i} \Delta_{i i}$, an ideal direct sum;

(iii) if each $I_{i}^{2} \supset \operatorname{Ann} I_{i}$, then $\Delta_{i j}$ is an abelian ideal of $\operatorname{Der}_{k} B$ 
when $i \neq j$;

(iv) if each $I_{i}^{2} \supset$ Ann $I_{i}$ and if each $\Delta_{i i}$ is a nilpotent Lie algebra, then $\operatorname{Der}_{k} B$ is a nilpotent Lie algebra;

(v) in particular, if all derivations of $I_{i}$ are nilpotent, then $I_{i}^{2} \supset$ Ann $I_{i}$, and all derivations of $B$ are nilpotent.

We now go on to construct nilpotent associative algebras which admit only nilpotent derivations. Choose integers $\alpha, \beta \geqq 3$ with $\alpha+$ $\beta-\alpha \beta$ not divisible by char $k$. Let $W$ be the $k$-algebra (without unity) on two generators $u, v$ satisfying the relations

$$
u v=0, \quad u^{\alpha}=v u=v^{\beta} .
$$

LEMMA 3.2. The k-algebra $W$ is finite-dimensional nilpotent associative with Ann $W \subset W^{2}$ and with all derivations nilpotent.

Proof. Only the nilpotence of $D \in \operatorname{Der}_{k} W$ needs to be checked. Since $W$ is nilpotent, it suffices to prove $D W \subset W^{2}$. We have, for $a, b, c, d \in k$,

$$
D u \equiv a u+b v\left(\bmod W^{2}\right), D v \equiv c u+d v\left(\bmod W^{2}\right) .
$$

Now one checks that $0=D(u v)$ forces $b=c=0$. Having this, one checks that $D u^{\alpha}=D(v u)=D v^{\beta}$ forces $\alpha a=a+d=\beta d$, whence $(\alpha+\beta-\alpha \beta) a=0$ in $k$. It follows that $a=d=0$, so that $D$ is nilpotent as asserted. Done.

In one part of his thesis, James Malley pushes these matters further by examining the structural consequences of the hypothesis $\operatorname{Der}_{k} N$ nilpotent [7, Chapter 5].

4. Proof of Theorem 2.1. First we construct $N$. Define $V_{0}=$ $W_{1} \oplus \cdots \oplus W_{t}$, where each $W_{i}$ is a copy of the algebra $W$ of Lemma 3.2 and $t=s$ if $s=1,2$ and $t=s-1$ if $s \geqq 3$. (These choices will be justified below.) The underlying $k$-space of $N$ is now defined to be $V_{0} \oplus V_{1} \oplus \cdots \oplus V_{s}$, and from now on we identify $V_{i}$ with the corresponding subspace of $N$.

We multiply in $N$ as follows: products in $V_{0}$ are as before, $V_{i} V_{j}=(0)$ for $i, j$ distinct and, for $x, y$ in $V_{i}$ with $i \geqq 1$, define $x y=$ $b_{i}(x, y) z_{i}$ where $z_{i}, \cdots, z_{s}$ will now be chosen. Let $w_{j}$ be a nonzero element of the (one-dimensional) Ann $W_{j}$. If $s=t=1$, define $z_{1}=$ $w_{1}$. If $s=t=2$, define $z_{1}=w_{1}+w_{2}$ and $z_{2}=w_{1}-w_{2}$. If $s \geqq 3$, define $z_{1}=w_{1}, \cdots, z_{s-1}=w_{s-1}$, and $z_{s}=w_{1}+\cdots+w_{s-1}$. We observe that $N$ is nilpotent, that $N^{2}=\left(V_{0}\right)^{2}$, and that Ann $N$ has $k$-basis $w_{1}, \cdots, w_{t}$. 
4.1. Now we check that $N$ is indecomposable (assertion (i)). If $s=1$, this is immediate because every nonzero ideal contains $z_{1}$. Assume in general that $N$ is an ideal direct sum $P \oplus Q$. On the one hand, we have Ann $N=P_{0} \oplus Q_{0}$, where $P_{0}=P \cap \operatorname{Ann} N$ and likewise for $Q_{0}$. On the other hand, the nondegeneracy of the bilinear form $b_{i}$ implies that $z_{i}$ is in either $P_{0}$ or $Q_{0}$. (In the anomalous case $s=t=2$, one also readily verifies that each $w_{i}$ is in either $P_{0}$ or $Q_{0}$.) Having this, and noting that any $t$ or fewer of the $z_{i}$ are linearly independent, one sees that the equation $t=\operatorname{dim} \operatorname{Ann} N=$ $\operatorname{dim} P_{0}+\operatorname{dim} Q_{0}$ forces $Q_{0}$ (say) to be zero, whence $Q=(0)$. Thus $N$ is indecomposable, as asserted.

Comment. Our choice of $t$ was complicated by the possibility that the scalar field $k$ is finite and "small". For $k$ infinite and $s \geqq$ 2 , taking $t=2$ suffices.

Now we analyze $\operatorname{Der}_{k} N$. For $i=0,1, \cdots, s$, let $\pi_{i}: N \rightarrow N$ be the usual projection on the subspace $V_{i}$ corresponding to the decomposition $N=V_{0} \oplus \cdots \oplus V_{s}$. In what follows, we take $D$ in $\operatorname{Der}_{k} N$ and define $D_{i j}=\pi_{i} \circ D \circ \pi_{j}$, a $k$-linear map (not a priori a derivation) $N \rightarrow$ $N$, so that $D=\sum_{0 \leqq i, j \leqq s} D_{i j}$.

4.2. For $1 \leqq i \leqq s$, we prove $D_{i 0}\left(N^{2}\right)=(0)$. Since $N^{2}=\left(V_{0}\right)^{2}$, it suffices to consider $x, y \in V_{0}$. Then $D_{i 0}(x y)=D(x y)-\sum_{j \neq i} D_{j 0}(x y)$. But the left hand side here is in $V_{i}$ and $D(x y)$ is in $V_{0}$. Thus the right hand side has zero projection into $V_{i}$. Thus both sides are zero.

4.3. We prove that the restriction of $D_{00}$ to $V_{0}$ is a derivation (and therefore nilpotent by (3.1) and (3.2)). For let $x, y \in V_{0}$. Then $D_{00}(x y)=D(x y)-\sum_{i \geqq 1} D_{i 0}(x y)$, and the result follows from (4.2) and the fact that $V_{0} V_{i}=V_{i} V_{0}=(0)$ for $i \neq 0$.

4.4. We prove that if $1 \leqq i<j \leqq s$, then $D_{i j}=D_{j i}=0$. For let $x \in V_{i}, y \in V_{j}$. Then

$$
0=D(0)=D(x y)=\left(D_{j i} x\right) y+x D\left({ }_{i j} y\right)=b_{j}\left(D_{j i} x, y\right) z_{j}+b_{i}\left(x, D_{i j} y\right) z_{i} .
$$

Since the $z_{i}$ are pairwise linearly independent (consider our definition for $s=2)$, we have $b_{j}\left(D_{j i} x, y\right)=b_{i}\left(x, D_{i j} y\right)=0$. But if $D_{j i} x \neq 0$, then the nondegeneracy of $b_{j}$ implies there exists $y$ in $V_{j}$ such that $b_{j}\left(D_{j_{i}} x\right.$, $y) \neq 0$. Thus $D_{j i}=0$, and likewise for $D_{i j}$ by symmetry.

4.5. We prove that if $1 \leqq i \leqq s$, then $b_{i}\left(D_{i i} x, y\right)+b_{i}\left(x, D_{i i} y\right)=0$ for all $x, y$ in $V_{i}$. For consider $D(x y)=b_{i}(x, y) D z_{i}=b_{i}(x, y) D_{00} z_{i}$, by (4.2). On the other hand, 


$$
D(x y)=x\left(D_{i i} y\right)+\left(D_{i i} x\right) y=\left\{b_{i}\left(x, D_{i i} y\right)+b_{i}\left(D_{i i} x, y\right)\right\} z_{i} .
$$

If this last coefficient is nonzero, then $z_{i}$ is an eigenvector for $D_{00}$ whose corresponding eigenvalue is nonzero, an impossibility by (4.3).

4.6. We prove that, for $1 \leqq i \leqq s, D_{i i}$ is a derivation of $N$. For if $x, y \in N$, then on the one hand $x y \in\left(V_{0}\right)^{2}$, so that $D_{i i}(x y)=0$. On the other hand, $x\left(D_{i i} y\right)+\left(D_{i i} x\right) y=\pi_{i}(x) D_{i i}\left(\pi_{i} y\right)+\left(D_{i i} \pi_{i}(x)\right) \pi_{i}(y)=$ 0 by (4.5). This settles (4.6).

4.7. For $D \in \operatorname{Der}_{k} N$, we define $D^{\sharp}=D-\sum_{1 \leqq i \leqq s} D_{i i}$, a derivation of $N$ by (4.6). Thus we have a vector space decomposition $\operatorname{Der}_{k} N=$ $\Lambda_{1} \oplus \cdots \oplus \Lambda_{s} \oplus \Omega$, where $\Lambda_{i}=\left\{D \in \operatorname{Der}_{k} N \mid D=D_{i i}\right\}$ and $\Omega=\{D \in$ $\left.\operatorname{Der}_{k} N \mid D=D^{\sharp}\right\}$. It is immediate that $\Lambda_{i}$ is a Lie subalgebra of $\operatorname{Der}_{k} N$.

4.8. Now we prove that $\Lambda_{i} \cong o\left(V_{i}, b_{i}\right)$ for $1 \leqq i \leqq s$ (statement (iii)). By (4.5) we have an embedding of $\Lambda_{i}$ into $o\left(V_{i}, b_{i}\right)$. To see that this embedding is surjective, let $f \in o\left(V_{1}, b_{1}\right)$ and extend $f$ to $\hat{f}: N \rightarrow$ $N$ by defining $\hat{f}\left(V_{j}\right)=0$ for $j \neq i$. One readily checks that $\hat{f}$ is a derivation of $N$, whence $A_{i} \cong o\left(V_{i}, b_{i}\right)$.

4.9. We give a direct proof that $\Omega$ is a Lie ideal in $\operatorname{Der}_{k} N$. Let $D \in \Omega, E \in \operatorname{Der}_{k} N$. We will show $(D E)_{i i}=(E D)_{i i}=0$ for each $i \geqq 1$. Now $(D E)_{i i}=\sum_{0 \leqq j \leqq s} D_{i j} E_{j i}=D_{i 0} E_{0 i}$ and, likewise, $(E D)_{i i}=$ $E_{i 0} D_{0 i}$. Since $D_{i 0}\left(N^{2}\right)=E_{i 0}\left(N^{2}\right)=(0)$ by $(4.2)$, we are done if we can prove that for any derivation $E$ (say), $E_{0 i}(N) \subset N^{2}$. Now note that $N^{2}=\left(V_{0}\right)^{2} \supset($ Ann $N: N)=\{u \in N \mid u N, N u \subset$ Ann $N\}$. Thus, one takes $x_{i} \in V_{i}, x_{0} \in V_{0}$ and readily checks that $x_{0} E_{0 i}\left(x_{i}\right)$ and $E_{0 i}\left(x_{i}\right) x_{0}$ are in Ann $N$.

4.10. To prove statement (iv) that $D \in \Omega$ is nilpotent (whence the ideal $\Omega$ is nilpotent by Engel's theorem), write $D=\sum_{i} D_{i 0}+D_{00}+$ $\sum_{i} D_{0 i}$ with $1 \leqq i \leqq s$ and simplify the iterates $D^{2}, D^{3}, \ldots$ by applying these facts: $D_{00}$ is nilpotent, $D_{00}$ stablizes $N^{2}, D_{i 0}\left(N^{2}\right)=(0)$, in particular $D_{i 0} D_{0 i}=0$, and $D_{0 i}(N) \subset N^{2}$, as in (4.9). One readily sees that some power of $D$ vanishes. This completes the proof of Theorem 2.1.

5. Proof of Theorem 2.2. Define $N$ to be $P \oplus Q$ as follows. Use Theorem 2.1 to form a nilpotent $P$ such that $\operatorname{Der}_{k} P=\Lambda_{p}+\Omega_{p}$ and $A_{p}$ is a direct sum of the given algebras $o\left(V_{i}, b_{i}\right)$. The algebra $Q$ will be a "block strictly upper triangular" matrix algebra $T_{0}(\rho, k)$ which we will now discuss. 
Let $\rho=\left(r_{1}, \cdots, r_{m}\right)$ be a vector whose entries are positive integers, and let $r=r_{1}+\cdots+r_{m}$. Form the usual full $r$ by $r$ matrix algebra $M(r, k)$ and imagine each matrix partitioned into rectangular blocks of size $r_{i}$ by $r_{j}$ as usual, so that reading down the "block main diagonal" one sees blocks of size $r_{1}$ by $r_{1}, r_{2}$ by $r_{2}, \cdots, r_{m}$ by $r_{m}$. The "block upper triangular" subalgebra $T(\rho, k)$ of $M(r, k)$ consists of all matrices with only zero blocks below the block main diagonal, while the "block strictly upper triangular" algebra $T_{0}(\rho, k)$ is the nilpotent subalgebra of $T(\rho, k)$ consisting of all matrices with only zero blocks both below and on the block main diagonal. One checks that $T_{0}(\rho, k)=\operatorname{rad} T(\rho, k)$ and that permuting the entries of the vector $\rho$ may yield nonisomorphic algebras.

The content of the following routine exercise is that all derivations of $T_{0}(\rho, k)$ are induced by bracketing with elements from $T(\rho, k)$.

LEMmA 5.1. There is a short exact sequence of Lie algebra homomorphisms

$$
0 \longrightarrow k \cdot 1+\operatorname{Ann} T_{0}(\rho, k) \stackrel{c}{\longrightarrow} T(\rho, k)_{\text {Lie }} \stackrel{\delta}{\longrightarrow} \operatorname{Der}_{k} T_{0}(\rho, k) \longrightarrow 0,
$$

where $\iota=$ inclusion and $(\delta c) c_{0}=\left[c, c_{0}\right]$.

We note some consequences for the derivations of $T_{0}(\rho, k)$. It is immediate that $T(\rho, k)_{\mathrm{Lie}} \cong\left(\bigoplus_{i} M\left(r_{i}, k\right)_{\mathrm{Lie}}\right)+T_{0}(\rho, k)_{\mathrm{Lie}}$, a semidirect sum. Moreover, if the characteristic of $k$ does not divide $r_{i}$, then each $M\left(r_{i}, k\right)_{\mathrm{Lie}}=s l\left(r_{i}, k\right) \oplus k e_{i}$ where $e_{i}$ is the identity of $M\left(r_{i}, k\right)$, $s l\left(r_{i}, k\right)$ consists of those matrices of trace zero, and this sum is Lie direct. Applying Lemma 5.1 we conclude, under the hypothesis on the characteristic of $k$, that the solvable radical of $\operatorname{Der}_{k} T_{0}(\rho, k)$ is itself a semidirect sum of the toroidal subalgebra $\sum_{i} k \delta e_{i}$ with the nilpotent ideal $\delta T_{0}(\rho, k)_{\text {Lie }}$. Note too that $\sum_{i} \delta e_{i}=\delta(1)=0$.

Having this general analysis of $T_{0}(\rho, k)$, we may complete the proof of Theorem 2.2. Given $r_{1}, \cdots, r_{n}$, in the statement, we define $\rho=\left(r_{1}, \cdots,\left\{r_{n}, 1,1\right)\right.$ and $Q=T_{0}(\rho, k)$. Note that $Q^{2} \supset$ Ann $Q$. It follows from the first paragraph of this section and from Lemma 3.1 that $\operatorname{Der}_{k} N=\operatorname{Der}_{k}(P \oplus Q)=\operatorname{Der}_{k} P \oplus \operatorname{Der}_{k} Q \oplus \Delta_{P Q} \oplus \Delta_{Q P}$ as a vector space, with $\Delta_{P Q}$ and $\Delta_{Q P}$ abelian ideals consisting of nilpotent derivations as in (3.1). Note $\left[\Delta_{P Q}, \Delta_{Q P}\right]=(0)$.

From Theorem 2.1, $\operatorname{Der}_{k} P=\Lambda_{P}+\Omega_{P}$ with $\Lambda_{P}$ a direct sum of specified $o\left(V_{i} b_{i}\right)$ and $\Omega_{P}$ an ideal of nilpotent derivations. Likewise, from Lemma 5.1, $\operatorname{Der}_{k} Q=\Lambda_{Q}+\Omega_{Q}$ where $\Lambda_{Q}$ is a direct sum of specified $s l\left(r_{i}, k\right)$ and $\Omega_{Q}=\left(\sum_{i=1}^{i=n+2} k \delta e_{i}\right)+\delta T_{0}(\rho, k)$. Note that the toroidal algebra spanned by the $\delta e_{i}$ has dimension $n+1$ (cf. statement (iii)). Theorem 2.2 follows by putting $\Lambda=\Lambda_{P} \oplus \Lambda_{Q}$ (cf. statement 
(ii)) and $\Omega=\Omega_{P}+\Omega_{Q}+\Delta_{P Q}+\Delta_{Q P}$.

\section{REFERENCES}

1. J. Dixmier and W. Lister, Derivations of nilpotent Lie algebras, Proc. Amer. Math. Soc., 8 (1957), 155-158.

2. F. J. Flanigan, Radical behavior and the Wedderburn family, Bull. Amer. Math. Soc., 79 (1973), 66-70.

3. - Radical embedding, genus, and toroidal derivations of nilpotent associative algebras, Bull. Amer. Math. Soc., 80 (1974), 986-990.

4. M. Hall, Jr., The position of the radical in an algebra, Trans. Amer. Math. Soc., 48 (1940), 381-404.

5. N. Jacobson, Lie Algebras, Interscience, New York, 1962.

6. G. Leger and E. Luks, On derivations and holomorphs of nilpotent Lie algebras, Nagoya Math. J., 44 (1971), 39-50.

7. J. Malley, Automorphisms and derivations of associative algebras, $\mathrm{Ph}$. D. thesis, University of California, San Diego, 1975.

Received June 18, 1974.

San Diego State University 


\section{PACIFIC JOURNAL OF MATHEMATICS}

\section{EDITORS}

RICHARD ARENS (Managing Editor)

University of California

Los Angeles, California 90024

\section{J. DugundJI}

Department of Mathematics University of Southern California Los Angeles, California 90007

D. Gilbarg and J. Milgram

Stanford University

Stanford, California 94305
University of Washington Seattle, Washington 98105

\section{ASSOCIATE EDITORS}
E. F. BECKENBACH
B. H. NeumanN
F. WolF
K. YoShIDA

\section{SUPPORTING INSTITUTIONS}

\author{
UNIVERSITY OF SOUTHERN CALIFORNIA \\ STANFORD UNIVERSITY \\ UNIVERSITY OF TOKYO \\ UNIVERSITY OF UTAH \\ WASHINGTON STATE UNIVERSITY \\ UNIVERSITY OF WASHINGTON \\ $\stackrel{*}{*} \stackrel{*}{*} \stackrel{*}{ }{ }^{*}$ AMERICAN MATHEMATICAL SOCIETY
}

The Supporting Institutions listed above contribute to the cost of publication of this Journal, but they are not owners or publishers and have no responsibility for its content or policies.

Mathematical papers intended for publication in the Pacific Journal of Mathematics should be in typed form or offset-reproduced, (not dittoed), double spaced with large margins. Underline Greek letters in red, German in green, and script in blue. The first paragraph or two must be capable of being used separately as a synopsis of the entire paper. Items of the bibliography should not be cited there unless absolutely necessary, in which case they must be identified by author and Journal, rather than by item number. Manuscripts, in triplicate, may be sent to any one of the editors. Please classify according to the scheme of Math. Reviews, Index to Vol. 39. All other communications should be addressed to the managing editor, or Elaine Barth, University of California, Los Angeles, California, 90024.

The Pacific Journal of Mathematics expects the author's institution to pay page charges, and reserves the right to delay publication for nonpayment of charges in case of financial emergency.

100 reprints are provided free for each article, only if page charges have been substantially paid. Additional copies may be obtained at cost in multiples of 50 .

The Pacific Journal of Mathematics is issued monthly as of January 1966. Regular subscription rate: $\$ 72.00$ a year (6 Vols., 12 issues). Special rate: $\$ 36.00$ a year to individual members of supporting institutions.

Subscriptions, orders for back numbers, and changes of address should be sent to Pacific Journal of Mathematics, 103 Highland Boulevard, Berkeley, California, 94708.

\section{PUBLISHED BY PACIFIC JOURNAL OF MATHEMATICS, A NON-PROFIT CORPORATION}

Printed at Kokusai Bunken Insatsusha (International Academic Printing Co., Ltd.), 270, 3-chome Totsuka-cho, Shinjuku-ku, Tokyo 160, Japan.

\section{Copyright (C) 1975 by Pacific Journal of Mathematics} Manufactured and first issued in Japan 


\section{Pacific Journal of Mathematics}

\section{Vol. 57, No. $2 \quad$ February, 1975}

Norman Larrabee Alling, On Cauchy's theorem for real algebraic curves with boundary .......

Daniel D. Anderson, A remark on the lattice of ideals of a Prüfer domain ..................

Dennis Neal Barr and Peter D. Miletta, A necessary and sufficient condition for uniqueness of

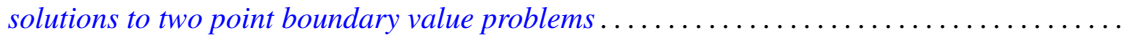

Ladislav Beran, On solvability of generalized orthomodular lattices . . . . . . . . . . ........

L. Carlitz, A three-term relation for some sums related to Dedekind sums . . . . . . . . . .....

Arthur Herbert Copeland, Jr. and Albert Oscar Shar, Images and pre-images of localization

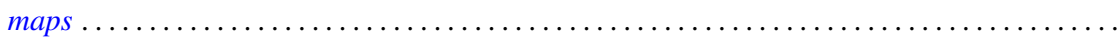

G. G. Dandapat, John L. Hunsucker and Carl Pomerance, Some new results on odd perfect

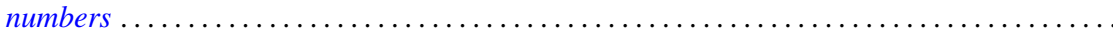

M. Edelstein and L. Keener, Characterizations of infinite-dimensional and nonreflexive

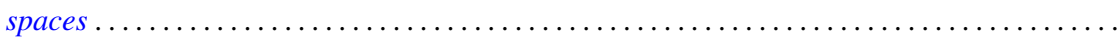

Francis James Flanigan, On Levi factors of derivation algebras and the radical embedding

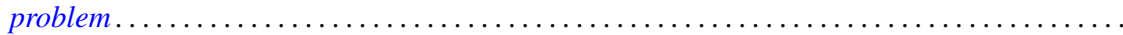

Harvey Friedman, Provable equality in primitive recursive arithmetic with and without

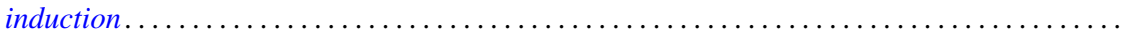

Joseph Braucher Fugate and Lee K. Mohler, The fixed point property for tree-like continua with

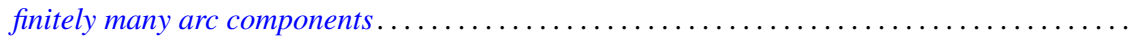

John Norman Ginsburg and Victor Harold Saks, Some applications of ultrafilters in

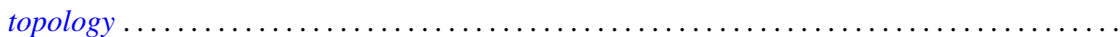

Arjun K. Gupta, Generalisation of a "square" functional equation .....................

Thomas Lee Hayden and Frank Jones Massey, Nonlinear holomorphic semigroups ..........

V. Kannan and Thekkedath Thrivikraman, Lattices of Hausdorff compactifications of a locally

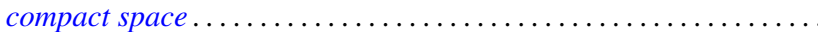

J. E. Kerlin and Wilfred Dennis Pepe, Norm decreasing homomorphisms between group

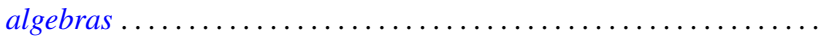

Young K. Kwon, Behavior of $\Phi$-bounded harmonic functions at the Wiener boundary ...

Richard Arthur Levaro, Projective quasi-coherent sheaves of modules .

Chung Lin, Rearranging Fourier transforms on groups...........................

David Lowell Lovelady, An asymptotic analysis of an odd order linear differential equation . . 4475

Jerry Malzan, On groups with a single involution .......................... 481

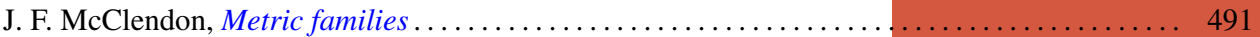

Carl Pomerance, On multiply perfect numbers with a special property .

Mohan S. Putcha and Adil Mohamed Yaqub, Polynomial constraints for finiteness of semisimple rings. .

Calvin R. Putnam, Hyponormal contractions and strong power convergence . . . . . . . . . 531

Douglas Conner Ravenel, Multiplicative operations in $\mathrm{BP} * \mathrm{BP} \ldots \ldots \ldots \ldots \ldots \ldots \ldots \ldots \ldots .539$

Judith Roitman, Attaining the spread at cardinals which are not strong limits . . . . . . . . . 545

Kazuyuki Saitô, Groups of *-automorphisms and invariant maps of von Neumann algebras . . . 553

Brian Kirkwood Schmidt, Homotopy invariance of contravariant functors acting on smooth

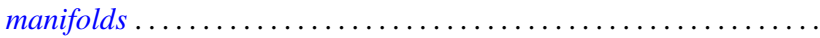

Kenneth Barry Stolarsky, The sum of the distances to $N$ points on a sphere.

Mark Lawrence Teply, Semiprime rings with the singular splitting property.

J. Pelham Thomas, Maximal connected Hausdorff spaces..............

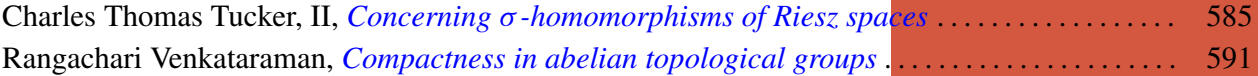

William Charles Waterhouse, Basically bounded functors and flat sheaves . . . . . . . . . . . 597

David Westreich, Bifurcation of operator equations with unbounded linearized part ......... 611

William Robin Zame, Extendibility, boundedness and sequential convergence in spaces of 